\title{
Editorial
}

\section{Workforce development: challenges for practice, professionalization and progress}

Workforce development stands out as a neglected focus of scholarship in the field of public health nutrition. So much so that it undermines the development of public health nutrition as a field of practice and professional activity, and ultimately limits the effectiveness of effort applied in this arena. This editorial challenges readers to consider and address ten key challenges (under the sub-headings below) relevant to public health nutrition workforce development. These preface a number of workforce development papers published in this issue from Canada, South Africa, the USA and Australia, which collectively should stimulate further debate, scholarship and the necessary focus on workforce development as a core function of public health nutrition effort, central to the ongoing professionalization of public health nutrition. Consistent with other areas of public health, a systems approach to workforce development is needed, which recognizes the relationships between the work, the worker and the work setting ${ }^{(1)}$ in the public health nutrition context.

\section{Workforce development research is required}

Workforce development should be considered like any other public health nutrition intervention in terms of developing, implementing and evaluating strategies with an emphasis on achieving sustainable and measurable outcomes. This requires an investment in research. Intelligence from published research specifically focusing on workforce development is limited to a number of countries (mostly developed economies). As a result, there are large gaps in the literature that limit strategic and intelligencebased workforce development ${ }^{(2)}$ in many part of the world where the need for workforce development is most apparent. Ironically it is likely that this is partly the result of limited investments in workforce development research by governments, who are also usually major employers.

\section{Agree on definitions for public health nutrition and be consistent when communicating with key stakeholders}

While employers and other key decision makers ask the question 'What is public health nutrition?', workforce development will continue to be an uphill battle. Consensus on definitions for public health nutrition as a disciplinary area, and conceptual clarity about what the field involves in terms of work, is critical. Fortunately, consensus on definitions for public health nutrition appears to have been galvanized in the definition adopted by the newly constituted World Public Health Nutrition Association, which defines public health nutrition as the promotion and maintenance of nutrition-related health and well-being of populations through the organized efforts and informed choices of society. This broad conceptualization of public health nutrition pre-dates and encapsulates the thrust of the New Nutrition Science Project ${ }^{(3)}$ and is not inconsistent with previously suggested alternatives such as 'Public Nutrition, (4). Consistent communication and marketing of public health nutrition as a field of practice to employers and other stakeholders is a primary challenge.

\section{Understand the work required}

Key stakeholders such as employers need to be able to understand what work is required in order to address public health nutrition issues, so that they can identify, engage and invest in practitioners to perform this work. Core functions statements such as those previously suggested (Table 1) codify the work involved and help focus work effort.

It is recognized that that the work of public health nutrition will vary depending on cultural, organizational, population and other contexts. The ongoing challenge is to describe, communicate and understand the work required in order to develop the capacity needed to perform this work.

\section{Focus on the determinants of workforce capacity}

Public health nutritionists will recognize the importance of determinant analysis as a basis for focusing and prioritizing strategies for intervention. This approach is applicable to workforce development, which should be considered like any other strategic initiative we apply in practice. Previous attempts to assess the determinants of workforce capacity ${ }^{(6)}$ have demonstrated that focusing on workforce size without attention to determinants such as workforce preparation, structure and composition, organization, management and practice improvement is likely to be ineffective. 
Table 1 Ten core functions for public health nutrition practice (practice functions) ${ }^{(5)}$

\begin{tabular}{|c|c|}
\hline & Core public health nutrition function \\
\hline Research and analysis & $\begin{array}{l}\text { 1. Monitor, assess and communicate population nutritional health needs and issues } \\
\text { 2. Develop and communicate intelligence }{ }^{\star} \text { about determinants of nutrition problems, policy impacts, } \\
\text { intervention effectiveness and prioritization through research and evaluation }\end{array}$ \\
\hline Build capacity & $\begin{array}{l}\text { 3. Develop the various tiers of the public health nutrition workforce and its collaborators through education, } \\
\text { disseminating intelligence* and ensuring organizational support } \\
\text { 4. Build community capacity and social capital to engage in, identify and build solutions to nutrition problems } \\
\text { and issues } \\
\text { 5. Build organizational capacity and systems to facilitate and coordinate effective public health nutrition action }\end{array}$ \\
\hline Intervention management & $\begin{array}{l}\text { 6. Plan, develop, implement and evaluate interventions that address the determinants of priority public health } \\
\text { nutrition issues and problems and promote equity } \\
\text { 7. Enhance and sustain population knowledge and awareness of healthful eating so that dietary choices are } \\
\text { informed choices } \\
\text { 8. Advocate for food and nutrition-related policy and government support to protect and promote health } \\
\text { 9. Promote, develop and support healthy growth and development throughout all life stages } \\
\text { 10. Promote equitable access to safe and healthy food so that healthy choices are easy choices }\end{array}$ \\
\hline
\end{tabular}

* Intelligence refers to information and knowledge from various sources that is used to inform decisions relating to problem resolution in public health nutrition practice.

\section{Multiple strategies are required}

There is often a tendency to limit considerations of workforce development strategies to training, in terms of workforce preparation via university courses and via post-employment continuing professional development. A failure to recognize and influence the other determinants of workforce capacity will constrain the impact of training and limit workforce development outcome attainment. The mantra of multi-strategy approaches so prevalent in public health practice is particularly important in the context of workforce development. Strategies that enhance practitioners' access to intelligence, mentoring programmes and workforce organization that increases interdisciplinary practice are needed irrespective of country, culture or priorities.

\section{Identify and support the structure of the public health nutrition workforce}

It should be widely accepted that the public health nutrition workforce is multidisciplinary and multi-tiered, and that workforce structures will vary between countries, health systems and other contexts. Identifying the key workforce constituents therefore is important in order to assess workforce development needs and focus workforce development efforts. This presents a major challenge, as the systems and research required to adequately monitor the size, structure and composition of the workforce contributing to public health nutrition effort are largely undeveloped. In different parts of the world it is difficult to clearly identify a public health nutrition workforce distinct from the broader public health workforce.

\section{Workforce development needs to be future- oriented and aspirational, and workforce development systems need to align}

Workforce development effort needs to focus on building capacity that will be required in the immediate- to medium-term future rather than what is needed now and based on current practice. This is partly because current practice may not be effective or appropriate and based on historical practice modes. The lag between supply (workforce preparation by the academic sector) and demand (employment opportunities, market needs, etc.) is as much as 5-10 years. This requires educators to base curriculum and pedagogy on competencies that reflect predicted and desirable practice needs (future-oriented rather than traditional). This is made difficult by the lack of scholarship in this area. Forward planning and developing systems to respond to changing and sudden increases in needs ('surge capacity') are major challenges for the public health nutrition workforce globally.

\section{Competencies should be considered the architecture for workforce development}

Competency frameworks are a valuable tool for workforce development because they codify the skills, knowledge and attitudes required for effective performance in the workplace. There is a growing consensus about the importance of, and general structure of, competencies required for effective public health nutrition practice, and that this agreement is evolving. Analysis of required competencies reinforces an argument that public health nutrition is a practice specialty requiring advancedlevel competencies that are developed in real-world practice contexts. Developing a specialist workforce tier, to lead and support public health nutrition action at a population level, is likely to be a priority and much needed workforce development strategy in most communities around the world. Specialization therefore is important, as is the competency architecture required to inform the development of specialists. Implicit in this emphasis on competencies is the importance of recognizing competencies as evolving standards that reflect the changing work of public health nutrition in response to new challenges. 


\section{Quality assurance: practice improvement and reorientation is required}

As stated earlier, the focus of workforce development should not dim once graduates enter the workforce but instead work to ensure that competencies developed can be efficiently applied to address and solve public health nutrition problems. Workforce quality assurance systems such as registration systems linked to continuing professional development and ethical practice are widely used across the health professions, and have been developed to support workforce development in public health nutrition. Many readers will be familiar with the development of a registration system for public health nutritionists by the Nutrition Society in the UK. Statutory registration is a feature of public health nutrition workforces such as those in Canada and the USA. The utility of, and need for, a global system of recognition and quality assurance for public health nutritionists will be important questions and challenges for the newly constituted World Public Health Nutrition Association.

\section{Dissemination, sharing and debate are needed}

The final challenge to readers is to participate in and encourage ongoing dialogue between academics, practitioners, communities and employers, to share intelligence relevant to effective capacity building via workforce development. Public health nutrition research and strategic intent has limited utility until it is effectively applied to improve public health. Practitioners are therefore critical capacity determinants for public health nutrition action. The nexus between academia and practice needs ongoing development, to enhance the effectiveness of the workforce and improve public health. Observations from both sides of the fence suggest we can all do this much better.

Public Health Nutrition is an ideal medium to facilitate this debate and dialogue, prompted in this issue by a number of workforce development papers from Canada, the USA, South Africa and Australia.

Roger Hughes Deputy Editor

\section{References}

1. Kennedy V \& Moore F (2001) A systems approach to public health workforce development. J Public Health Manag Pract 7, 17-22.

2. Hughes R (2003) A conceptual framework for intelligencebased public health nutrition workforce development. Public Health Nutr 6, 599-605.

3. Cannon G \& Leitzmann C (2005) The new nutrition science project. Public Health Nutr 8, 673-694.

4. Beaudry M \& Delisle H (2005) Public('s) nutrition. Public Health Nutr 8, 743-748.

5. Hughes R (2007) Practices overview. In Public Health Nutrition: From Principles to Practice, pp. 265-272 [M Lawrence and A Worsley, editors]. Crows Nest, NSW: Allen \& Unwin.

6. Hughes R (2006) A socio-ecological analysis of the determinants of national public health nutrition workforce capacity: Australia as a case study. Fam Community Health 29, 55-67.

\section{In this issue}

Workforce development is a major capacity-building strategy of great relevance to public health nutrition, yet little is published on this topic to inform policy, practice and future research. In this workforce development themed issue, a number of papers are presented that go some way to addressing this gap in scholarship and hopefully provide an impetus for more active dissemination of workforce development research and practice through this journal.

Fox et $_{\text {al. }}{ }^{(1)}$ present the results of a situational assessment of public health nutrition practice in Canada that has informed the development of a national workforce development strategy. This multifaceted study consisted of literature review, key informant interviews, a political, economic, environmental, social and technological (PEEST) factor analysis and a consensus meeting. Central to this approach has been the active engagement and participation of multiple stakeholders, which is critical to effective workforce development.

South of the border in the USA, which has arguably one of the world's largest and most developed public health nutrition workforces, Haughton and George ${ }^{(2)}$ describe the challenges for workforce development based on a long history and significant national investment in public health nutrition programmes. Key messages from this paper include the importance and recognition of the need for workforce monitoring and the dynamic nature of the workforce that needs to change in response to changing work environments and needs, particularly emphasizing the effect of changing government policy and resource allocation on influencing workforce practices. 